This item was submitted to Loughborough's Research Repository by the author.

Items in Figshare are protected by copyright, with all rights reserved, unless otherwise indicated.

\title{
Improvement of perceived vehicle performance through adaptive electronic throttle control
}

PLEASE CITE THE PUBLISHED VERSION

PUBLISHER

(C) IMechE / Professional Engineering Publishing

VERSION

VoR (Version of Record)

LICENCE

CC BY-NC-ND 4.0

\section{REPOSITORY RECORD}

Tuplin, Simon, Matt C. Best, and Martin A. Passmore. 2009. "Improvement of Perceived Vehicle Performance Through Adaptive Electronic Throttle Control”. figshare. https://hdl.handle.net/2134/4613. 
This item was submitted to Loughborough's Institutional Repository (https://dspace.lboro.ac.uk/) by the author and is made available under the following Creative Commons Licence conditions.

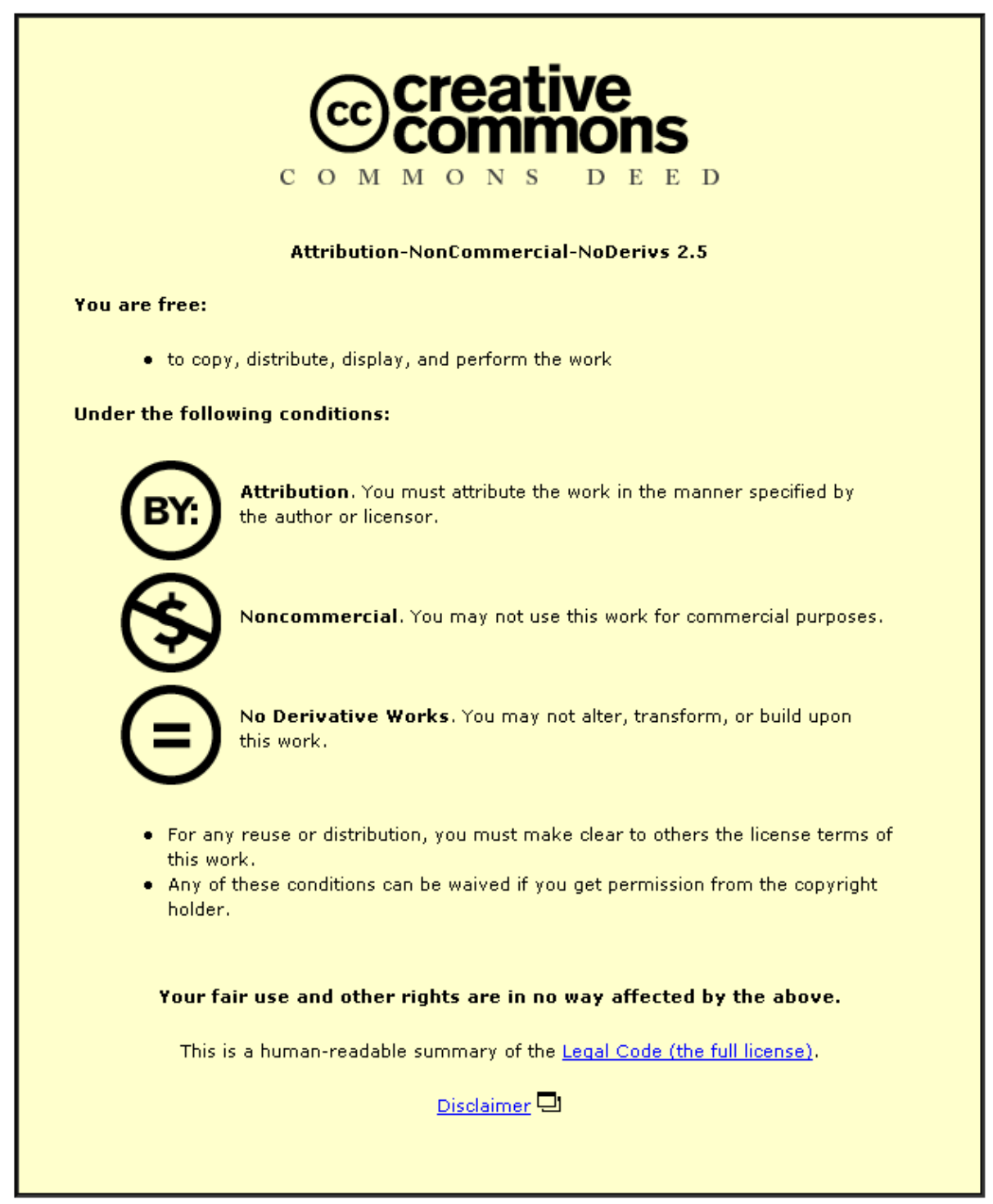

For the full text of this licence, please go to: http://creativecommons.org/licenses/by-nc-nd/2.5/ 


\title{
Improvement of perceived vehicle performance through adaptive electronic throttle control
}

S Tuplin*, M C Best and M A Passmore

Department of Aeronautical and Automotive Engineering, Loughborough University, Loughborough, UK

\begin{abstract}
With the advent of production electronic throttle control there is scope for increased customer satisfaction through the optimization of the throttle pedal demand map to individual drivers. The aim of this study is to develop algorithms to identify, from variables measured in real time on a test vehicle, the requirement for and the direction of adaptation of throttle pedal progression. An on-line appraisal procedure has been developed to identify the individual 'ideal' progression (IIP) for any driver. During the appraisal the subject is exposed to a series of pedal progressions, and their verbal response to each change is used to converge to their optimal setting. Vehicle data acquired on these appraisal drives have been regressed against IIP in a full factorial study, and the most statistically significant driver model established. A preliminary implementation of the model is used to demonstrate that throttle progression adapts appropriately towards IIP, thereby matching vehicle performance feel to driver expectations.
\end{abstract}

Keywords: adaptive throttle, drive appraisals, electronic throttle control, model regression, pedal progression, performance feel

NOTATION
$a, b, c, d$
$\hat{a}, \hat{b}, \hat{c}, \hat{d}$
ARMA
$b_{k}$
CBM
$\mathrm{d} T / \mathrm{d} D$
$\mathrm{~d} T / \mathrm{d} N$
$D$
$D_{t}$
$D_{t-1}$
$f_{1}, f_{2}, f_{3}, f_{4}$
$H(s)$
$i$
$\operatorname{IIP}$
$\operatorname{IIP}_{i}$
$j$
$k$

system coefficients

digital filter transformed coefficients

autoregressive moving average

subdivision of the range

(75th percentile algorithm)

customer behaviour model

rate of change in torque with pedal

demand

rate of change in torque with engine speed

pedal demand (per cent)

pedal demand at time $t$ (per cent)

pedal demand at time $t-1$ (per cent)

factorial parameters measured on the vehicle

general system

candidate rating number

individual ideal progression

concatenated $x$ for all candidates

test point

number of experiment
$M_{1}$

$M_{2}$

MRV

$n$

$n_{\mathrm{p}}$

$P_{\max }$

$P_{\text {MAX }}$

$P_{\text {min }}$

$P_{\text {MIN }}$

$Q_{3}$

$Q_{3 \mathrm{POSN}}$

$r$

$\mathbf{R}$

$\bar{R}_{\mathrm{w}} \quad$ mean Pearson correlation coefficients

$\bar{R}_{\text {wo }}$

The MS was received on 19 June 2002 and was accepted after revision for publication on 12 November 2002.

* Corresponding author: Department of Aeronautical and Automotive Engineering (AAE), Stewart Miller Building, West Park, Loughborough University, Ashby Road, Loughborough LE11 3TU, UK. with model regression variable $i$ included in the regression

slope of straight-line model, fitted below the model maximum slope of straight-line model, fitted above the model maximum model regression variable number of progression steps tested within a given iteration in the appraisal number of data points pedal gain (no units) maximum pedal gain (no units) within the appraisal maximum pedal gain (no units) minimum pedal gain (no units) within the appraisal minimum pedal gain (no units) 75th percentile position of the 75 th percentile candidate rating (unitary) returned Pearson error matrix (no units) mean Pearson correlation coefficients with model regression variable $i$ excluded from the regression poles of $H(s)$ signum function 


\begin{tabular}{|c|c|}
\hline$S_{j}$ & $\begin{array}{l}\text { candidate rating correlation score } \\
\text { (no units) }\end{array}$ \\
\hline SSV & steady state value \\
\hline$t$ & time $(\mathrm{s})$ \\
\hline $\mathbf{u}_{i}$ & $\begin{array}{l}\text { regression matrix for model regression } \\
\text { variable } k\end{array}$ \\
\hline$U(k)$ & $P$ input at iteration $k$ (no units) \\
\hline$U(k-1)$ & $P$ input at iteration $k-1$ (no units) \\
\hline WOT & wide-open throttle \\
\hline$x$ & $\begin{array}{l}\text { individual ideal progression vector, } \\
\text { of length } k\end{array}$ \\
\hline$x_{k}$ & model regression variable $k$ \\
\hline$y_{i}$ & individual ideal regression model \\
\hline$Y(k)$ & filter output at iteration $k$ (no units) \\
\hline$Y(k-1)$ & $\begin{array}{l}\text { filter output at iteration } k-1 \\
\text { (no units) }\end{array}$ \\
\hline $\mathrm{ZOH}$ & zero-order hold \\
\hline$\alpha, \beta, \gamma, \delta, \varepsilon$ & $\begin{array}{l}\text { least-squares coefficients of the } \\
\text { customer behaviour model }\end{array}$ \\
\hline$\alpha_{i}$ & $\begin{array}{l}\text { significance of model regression } \\
\text { variable } i\end{array}$ \\
\hline$\alpha_{k}$ & general least-squares fitted coefficients \\
\hline$\varepsilon$ & $\begin{array}{l}\text { unfitted error (least-squares } \\
\text { regression) }\end{array}$ \\
\hline$\lambda$ & range reduction factor (no units) \\
\hline
\end{tabular}

\section{INTRODUCTION}

Motor manufacturers continually strive to improve the product they offer to the motoring public. In the last two decades much of their effort has been directed towards identifying the precise needs and wishes of customers and then successfully transferring these to their product. One area to have received attention is the perceived performance of the vehicle. In this case the manufacturers have been limited to identifying the optimum set-up to satisfy all drivers. Passmore [1] and Passmore et al. [2] reported one such study. They carried out an extensive factorial study of performance feel, investigating factors, effects and interactions. The conclusions gained from the study are that, although actual wideopen throttle (WOT) acceleration is the primary parameter in giving good performance feel, secondary effects also have a powerful effect, and in particular the throttle progression (rate of change in torque with pedal demand, $\mathrm{d} T / \mathrm{d} D$ ) and rate of change in torque with engine speed, $\mathrm{d} T / \mathrm{d} N$.

The study identified the optimum set-up for throttle progression and $\mathrm{d} T / \mathrm{d} N$ but also showed a high variance in response from drivers and some evidence of demographic effects, which lead to the conclusion that performance feel benefits could be achieved if a vehicle's throttle system were able to adapt to the driver's preference. The advent of production electronic throttle control makes this a possibility and is the subject of this paper. This work details the development of an on-line adaptive throttle control system to meet these customer needs. At this stage a fully mapped system would be difficult to implement; therefore, to simplify the development, inference from Passmore [1] indicates that the first parameter to be considered in a one-dimensional system should be throttle progression. The primary aims of this study are to develop algorithms to identify, from parameters measured in real time on the vehicle, the requirement for and the direction of adaptation of the throttle progression and to implement these algorithms in a working system.

To implement such a system requires a model of driver satisfaction that can be evaluated in real time. To establish this 'customer behaviour model' (CBM) the individual ideal progression (IIP) for each driver needs to be identified. Thus a novel appraisal method has been developed to identify IIP accurately, using a series of iterative paired comparisons, implemented in an automated double-blind randomized block design process on the test vehicle. This method is described in section 2 of the paper. Measurements such as engine and vehicle speed are acquired during the appraisal, and a subset of these are linearly combined to generate a CBM model, described in section 3. Section 4 describes a statistical analysis to determine the most significant subset of variables to employ in the model, and the paper concludes with field trials of the final CBM, used to adapt progression in the test vehicle.

\section{IDENTIFICATION OF THE INDIVIDUAL IDEAL PROGRESSION (IIP): THE 'EYE TEST' METHOD}

The 'traditional' format of appraisal testing using, for example, the Loughborough Likert scale (cf. reference [1]), although well established and highly regarded, is inefficient at returning a reliable measure of IIP in a suitably short (less than $2 \mathrm{~h}$ ) time frame. Such methods require the driver to give ratings following each (typically fixed-length) test drive; this restricts the number of options that can be tested and relies on the fact that the driver has a good memory of what was good or bad. (It should be noted that the driver is unaware of the precise nature of the vehicle performance modification that is being made during the appraisal.) The goal here is to achieve testing which:

(a) exposes the driver to a wide range of operating conditions (progressions),

(b) identifies the range of progressions over which a given driver is insensitive to change,

(c) can be carried out over a single test drive, within the concentration span of the driver (ideally no more than $1 \mathrm{~h}$ ), 
(d) is robust to the limitations of the driver's rating consistency,

(e) is robust to 'incorrect' responses caused by changing environmental conditions and

(f) does not follow predictable patterns of change which might bias the driver's expectations and hence responses.

The solution described here uses paired comparisons to limit the concentration and rating skills required by the driver. The candidates are asked to state their preference to the current progression, giving an answer of better, same or worse than the previous setting. Also, the driver is allowed as much or as little time as he/she requires to make the assessment; this has risks in terms of (e) above but reaps considerably greater benefits in terms of (a) to (d). A degree of randomness in progression selection, together with automated (and hence 'double-blind') testing then ensures that (f) is satisfied.

\subsection{Detailed appraisal methodology}

Progression in this study is defined as an application of gain to the input-output relationship of the throttle pedal; thus

Throttle cable travel $=P \times$ pedal position

Initially, prototyping was carried out to identify the safe limits of progression over which the 'eye test' could operate. These limits were found by taking the test vehicle out, and changing the progression until the vehicle became undrivable:

\section{Lower limit $P_{\text {MIN }}=0.5$}

Upper limit $P_{\mathrm{MAX}}=3.5$

The lower limit might be justified even though it restricts maximum throttle opening to half WOT. It has been observed that some drivers do not even use full pedal travel in a conventional $(P=1)$ situation, indicating that they do not wish to use the full power of the engine, at least during the (B class road) driving environment tested. The lower limit maximizes controllability but, below this progression peak, torque is reduced to the point where the car is undrivable. The upper limit is defined by the point beyond which the car becomes uncontrollable, due to the very small amount of pedal travel to WOT. Gains of $P>1$ are saturated at WOT.

An initial range is selected within these limits, which covers most of the 'expected' IIPs as determined in previous appraisals. $n$ individual test points are then defined in this range by dividing it into $n-1$ equally spaced steps.

The process is then to record a rating between each pair of adjacent points, while attempting to minimize the predictability of the changes. This is achieved by randomly setting $n$ as either $n=5$ or $n=7$ and then subdividing the range into two. The order of execution of each half, and the direction of travel (increasing or decreasing $P$ ) with each half is also randomized. This is illustrated in the example shown in Fig. 1. The order of tests is shown by 1 to $\underline{8}$; therefore the ratings are recorded in the order a to f. (The second half of the range is tested first with increasing $P$, followed by the first half with decreasing $P$.) Note that, although a rating is requested between all changes in progression, the comparison between tests 4 and 5 is ignored, as is that between test $\underline{8}$ and the first test in the next iteration of the algorithm. Further randomness in test ordering is avoided to limit the number of these unproductive comparisons. Each of the ratings is recorded as follows:

$$
\begin{aligned}
& r=+1: \text { better than the last setting } \\
& r=0: \text { the same, or insignificant change compared } \\
& \quad \text { with the last setting } \\
& r=-1: \text { worse than the last setting }
\end{aligned}
$$

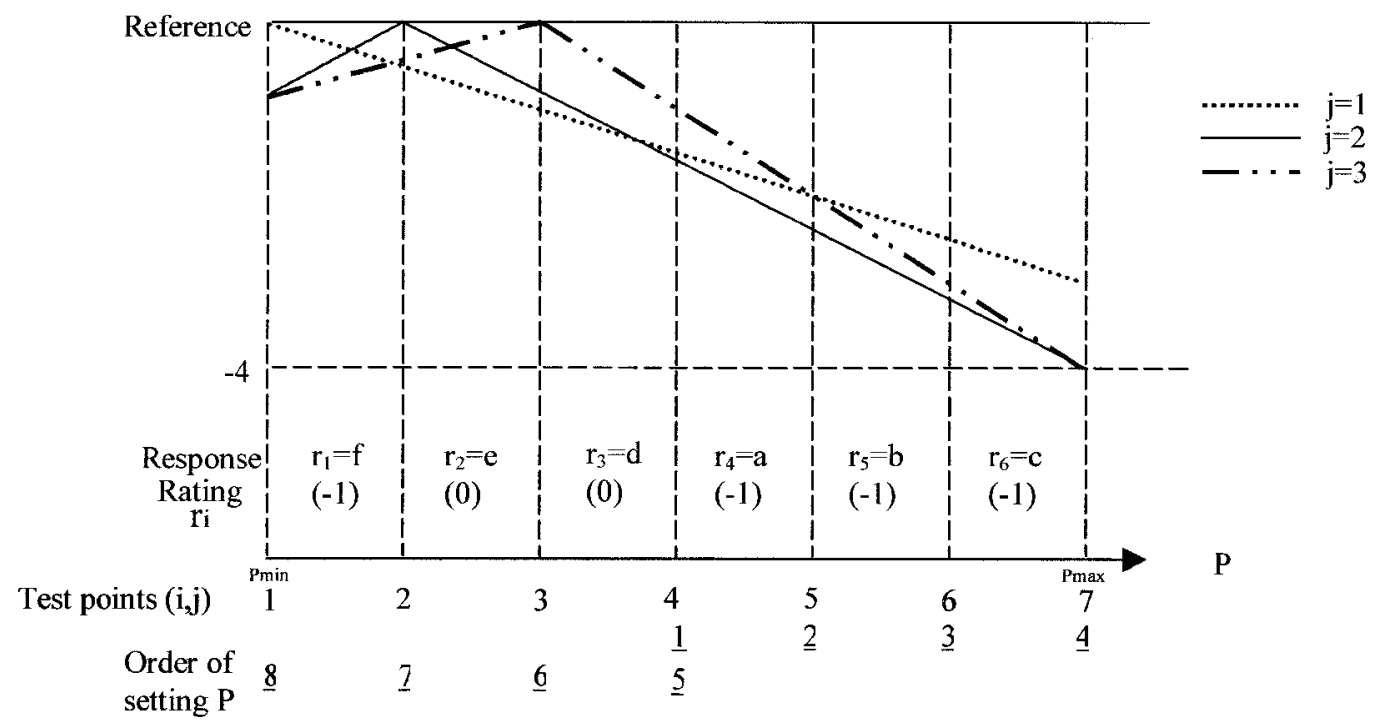

Fig. 1 Example of eye test slope estimation 
Using these ratings, a piecewise linear model is fitted about each of the $n$ tested progressions in turn, in order to locate the maximum (or exceptionally, the minimum) point of preference (see Fig. 1). For each candidate model, the peak is assumed to be at the $j$ th point, and slopes are calculated about that point. The slope of the first line is determined from the ratings $r_{i}$ as

$$
M_{1(j)}=\frac{\sum_{i=1}^{j^{-}} 1 r_{i} / \Delta P_{i}}{j-1}
$$

where

$$
\begin{aligned}
& \Delta P_{i}=P_{i+1}-P_{i} \text { if the test order has } P \text { increasing } \\
& \Delta P_{i}=P_{i}-P_{i+1} \text { if the test order has } P \text { decreasing }
\end{aligned}
$$

The second slope is then given as:

$$
M_{2(j)}=\frac{\sum_{i=j}^{n-1} r_{i} / \Delta P_{i}}{n-j}
$$

Note that $M_{1}$ is not calculated when $j=1$, and $M_{2}$ is omitted when $j=n$.

The optimal model is then selected as that which best fits all ratings, according to a correlation score $S_{j}$ :

$$
S_{j}=\sum_{i=1}^{j-1} r_{i} \operatorname{sgn}\left(\Delta P_{i} M_{1(j)}\right)+\sum_{i=j}^{n-1} r_{i} \operatorname{sgn}\left(\Delta P_{i} M_{2(j)}\right)
$$

Figure 1 also illustrates the line fitting defined by this process for the first three values of $j$; by inspection of the $r_{i}$, the best fit should be obtained at either $j=2$ or $j=3$, and this is confirmed by the correlation scores $S_{1}=3, S_{2}=S_{3}=5$. In cases such as this, where two scores are equal, the point closest to the midpoint of the range is taken ( $j=3$ in this case).

When the optimum point of the current range has been found, the process above is iterated, with the next set of tests centred on this optimum, and the range reduced by a factor $\lambda$, defined according to the correlation score achieved:

$$
\lambda=1-0.5 \frac{\max \left(S_{j}\right)}{\sum_{i=1}^{n^{-1}}\left|r_{i}\right|}
$$

Thus the search converges if consistent ratings are given.

Obvious adjustments are made to keep the testing within the bounds $P_{\text {MIN }} \leqslant P \leqslant P_{\text {MAX }}$ and the one exception to the rates given above is where the gradient search finds a minimum $\left(M_{1}<0\right.$ and $\left.M_{2}>0\right)$. This result indicates very poor driver consistency but, if it occurs, testing continues by centring the new range on $j=1$ or $j=n$ depending on which of $M_{1}$ or $M_{2}$ is best correlated, according to the appropriate single term of $S$ in equation (4). The candidate drives repeated iterations of this process until convergence is indicated by a consistent set of $r_{i}=0 \forall_{i}$.

In Fig. 2, the eye-test method is illustrated in simulation. A polynomial curve was used to represent IIP (peaking at $P=0.916)$ and to choose $r_{i}$ values $\left(r_{i}=0\right.$ where the change in the polynomial is less than 1$)$. With completely consistent responses (Fig. 2a) the eye test predicts that $P=0.916$, but the algorithm also performs very well in the face of significant disturbance. Figure $2 b$ shows a test with 12 of the $26 r_{i}$ randomly (and wrongly) chosen, and the final result is $P=0.899$.

In field trials the eye test appears to be successful, with all candidates saying that the final vehicle response was an improvement over the standard setting. Unexpectedly the general trend was that, the more subjectively aggressive the driver, the lower is the IIP that was preferred. This indicates that a more aggressive driver prefers (or requires) more control over the available power.

\section{(a) with 26 consistent responses}

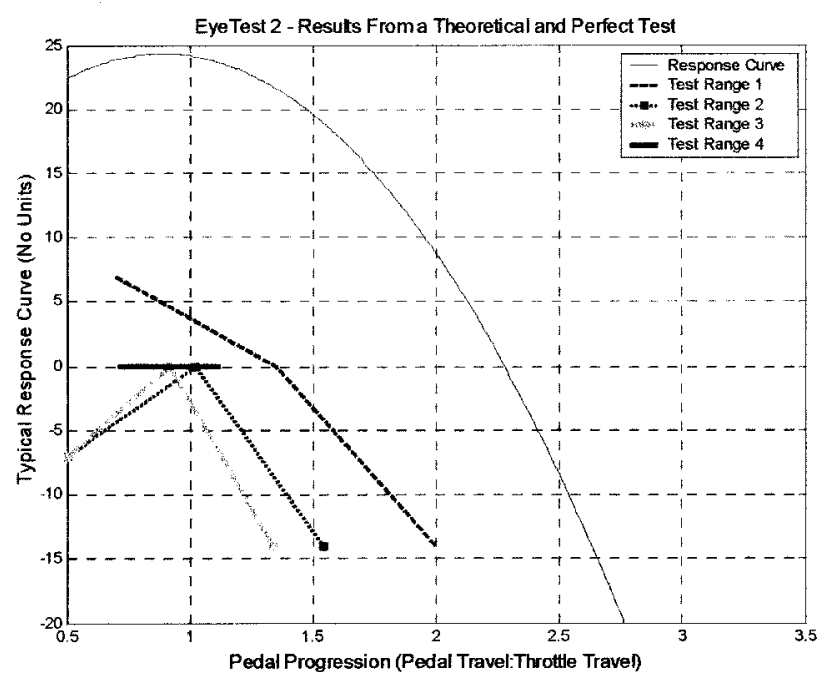

\section{(b) with $12 / 26$ inconsistent responses}

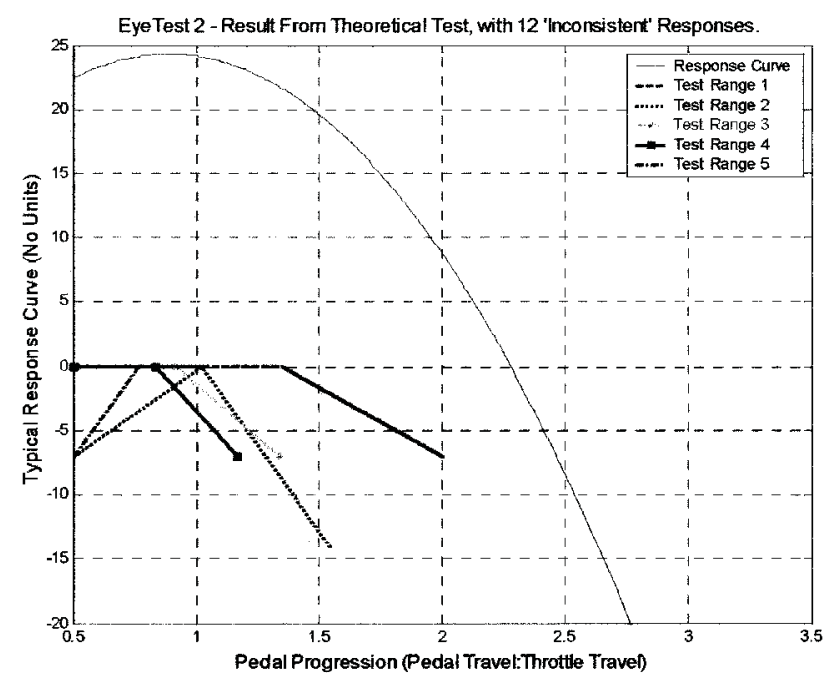

Fig. 2 Eye test simulation results: testing robustness 


\section{DEVELOPMENT OF A CUSTOMER BEHAVIOUR MODEL (CBM)}

Once the IIPs for a sample have been identified, a method is needed to correlate these with the driver's behaviour in so far as this can be measured in the vehicle. The behaviour model is constructed from variables sampled in real time on the vehicle, and from filtered variants of these. The 'eye-test' appraisal has a 'free driving format' [2], driven over predetermined B class roads using the Ford Focus 2.01 test vehicle. For this reason, data sampled from the vehicle during the appraisal can be used to correlate with the final IIP found. These data are ideal for two reasons:

1. They properly reflect the driver's mood during the appraisal; this is known to have an influence on IIP.

2. A wide range of different progressions are being executed throughout the data; therefore the influence of progression on the variables measured is minimal.

Table 1 illustrates the raw data acquired during the tests. From these, a relatively slow ARMA is also deduced (having a settling time of around $1200 \mathrm{~s}$ ), and a 75 th percentile measure $Q_{3}$ is also derived, using a memory and processor efficient algorithm which is described in the Appendix.

Note that the pedal rate is calculated using a backward-difference approximation, from pedal demand D:

$$
\frac{\mathrm{d} D}{\mathrm{~d} t}=\frac{D_{t}-D_{t-1}}{t_{t}-t_{t-1}}
$$

Seven raw data signals are thus derived from just four sensors, and these are manipulated to provide 28 different continuous variables, giving both rapidly and slowly varying measures of driving style. These $28 \mathrm{MRVs}$ can be united in various combinations in an attempt to provide a model for IIP, using multiple linear regression. The regression is carried out using a least-squares fit, returning a model of the form shown in the following equation:

$$
y=\hat{\alpha}_{0}+\hat{\alpha}_{1} x_{1}+\hat{\alpha}_{2} x_{2}+\cdots+\hat{\alpha}_{k} x_{k}+\varepsilon
$$

where

$$
\begin{aligned}
& y=\text { IIP } \\
& \alpha_{k}=\text { least-squares fitted coefficients } \\
& x_{k}=\mathrm{MRV}_{i} \\
& \varepsilon=\text { unfitted error }
\end{aligned}
$$

Figure 3 shows a sample regression using three MRVs. There are 12 candidates represented in the plot, with the $x$ axis relating to the time on a test drive. Each candidate has an equal amount of time represented.

Figure 3 indicates that distinct differences exist between drivers, demonstrating that a suitable customer model might be defined. Thus a statistical experiment was conducted to identify an accurate CBM, using a small number of MRVs.

\section{STATISTICAL ANALYSIS TO DETERMINE THE BEST MODEL}

A factorial experiment is a well-known applied statistical method used to consider all possible combinations of variables in a multiple linear regression model. To cover all possible combinations of all variables, $2^{k}$ tests would be performed. As 28 variables have been defined for testing, $2^{28}$ tests would need to be performed. As this would take approximately 466 days, a more efficient

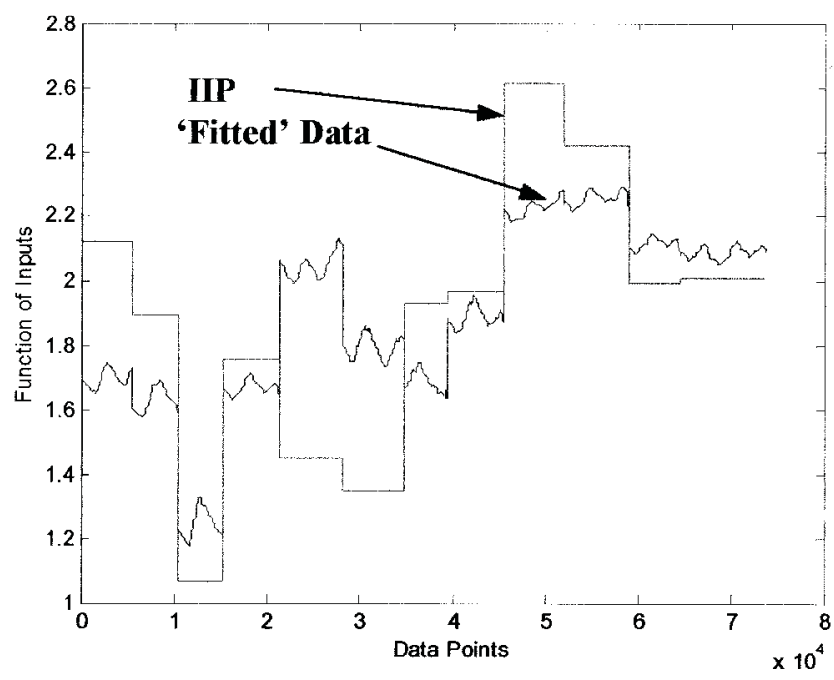

Fig. 3 Sample regression of MRVs to IIP

Table 1 Model regression variables (MRVs)

\begin{tabular}{lllll}
\hline Global variable & Raw signal & ARMA* & $Q_{3}$ & $Q_{3}$ ARMA $^{*}$ \\
\hline Engine speed & $\times$ & $\times$ & $\times$ & $\times$ \\
Engine speed at gear change-up point & $\times$ & $\times$ & $\mathrm{ZOH}^{\dagger}$ (not $\left.Q_{3}\right)$ & $\mathrm{ZOH} \mathrm{ARMA}^{* \dagger}$ \\
Absolute lateral acceleration & $\times$ & $\times$ & $\times$ & $\times$ \\
Longitudinal acceleration & $\times$ & $\times$ & $\times$ & $\times$ \\
Throttle pedal position & $\times$ & $\times$ & $\times$ & $\times$ \\
Throttle pedal rate & $\times$ & $\times$ & $\times$ & $\times$ \\
Vehicle speed & $\times$ & $\times$ & $\times$ & $\times$ \\
\hline
\end{tabular}

* ARMA, autoregressive moving average.

$\dagger \mathrm{ZOH}$, zero-order hold. 
design was needed! The method adopted was to randomly split the variables into two 14-variable factorial tests. From these two separate experiments the 14 most significant variables were then tested in a third factorial experiment and the results of this experiment used to produce the final model.

For each regression in the factorial experiment a Pearson correlation coefficient is calculated measuring the extent of the linear fit of the model; thus the factorial experiment has the form

$$
[\mathbf{R}]=\left[\frac{n_{\mathrm{p}} \sum \boldsymbol{x}\left(\mathbf{u}_{i} \boldsymbol{y}_{i}\right)-\sum \boldsymbol{x} \sum \mathbf{u}_{i} \boldsymbol{y}_{i}}{\sqrt{\left[n_{\mathrm{p}} \sum \boldsymbol{x}^{2}-\left(\sum \boldsymbol{x}\right)^{2}\right]\left[n_{\mathrm{p}} \sum\left(\mathbf{u}_{i} \boldsymbol{y}_{i}\right)^{2}-\left(\sum \mathbf{u}_{i} \boldsymbol{y}_{i}\right)^{2}\right]}}\right]_{i=1}^{i=k}
$$

where

$$
\begin{aligned}
\boldsymbol{y}_{i} & =\left(\mathbf{u}_{i}^{\mathrm{T}} \mathbf{u}_{i}\right)^{-1} \mathbf{u}_{i}^{\mathrm{T}} \boldsymbol{x} \\
\mathbf{u}_{i} & =\left[\begin{array}{cccc}
1 & x_{1}\left(t_{0}\right) & \cdots & x_{i}\left(t_{0}\right) \\
\vdots & \vdots & \vdots & \vdots \\
1 & x_{1}\left(t_{n}\right) & \cdots & x_{i}\left(t_{n}\right)
\end{array}\right] \\
\boldsymbol{x} & =\left[\begin{array}{c}
\mathrm{IIP}_{1} \\
\vdots \\
\operatorname{IIP}_{i}
\end{array}\right], \quad \operatorname{IIP}_{i}=\left[\begin{array}{c}
\operatorname{IIP}_{n_{\mathrm{p}} i} \\
\vdots \\
\operatorname{IIP}_{n_{\mathrm{p}} i}
\end{array}\right]_{n_{\mathrm{p}}=1}^{n_{\mathrm{p}}=k}
\end{aligned}
$$

where

$$
\begin{aligned}
& k=\text { number of experiments } \\
& t_{n}=\text { datum at time } t \\
& \mathbf{R}=\text { returned error matrix } \\
& n_{\mathrm{p}}=\text { number of data points }
\end{aligned}
$$

This was evaluated for $k=2^{14}$ experiments covering all possible combinations of half of the MRVs. The returned error matrix $\mathbf{R}$ can now be analysed to identify the most significant model. The significance of an individual MRV was calculated using

$$
\alpha_{i}=\bar{R}_{\mathrm{w}}-\bar{R}_{\mathrm{wo}}
$$

where

$$
\begin{aligned}
& \alpha_{i}=\text { significance of } \mathrm{MRV}_{i} \\
& \bar{R}_{\mathrm{w}}=\text { mean Pearson correlation coefficients with } \\
& \quad \mathrm{MRV}_{i} \text { included in the regression } \\
& \bar{R}_{\mathrm{wo}}=\text { mean Pearson correlation coefficients with } \\
& \quad \mathrm{MRV}_{i} \text { excluded from the regression }
\end{aligned}
$$

If none of the MRVs is statistically significant in providing the 'best' model, the significance values $\alpha_{i}$ will be randomly distributed. This can be clearly illustrated using the convenient graphical technique of normal score plotting, where in the case of purely random data the normal plot approximates a straight-line graph, whose slope is defined by the standard deviation (see, for example, reference [3]). Conversely, statistically significant MRVs appear as deviations from this straight line, and the degree of deviation illustrates the level of significance.

Figure 4 shows the results for single-variable regressions from the two initial factorial experiments. Eight of the MRVs have positive significance which is above the 'noise' level; these are (coincidentally) the values with a normal score greater than 0.5 . Of these, three have major significance.

The eight positively significant MRVs were then combined with six other, randomly selected MRVs, to conduct a final factorial experiment. Figure 5 shows the results, and here the significance values of all possible combinations of up to four MRVs are illustrated.

As can be seen, there is obvious deviation from the straight line, with several combinations of four MRVs being statistically significant. Of these, all should provide good models of customer behaviour but, from an operational point of view, only those that have a large

\section{(a) Factorial Experiment 1}

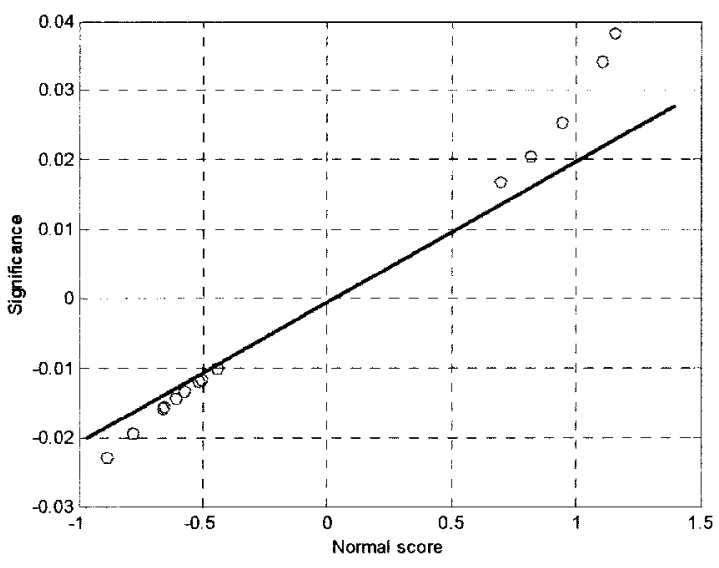

(b) Factorial Experiment 2

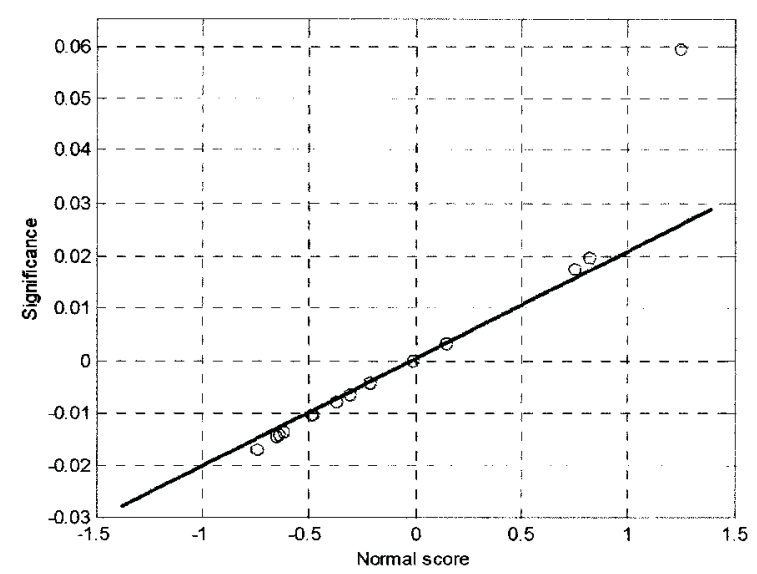

Fig. 4 Initial factorial experiment results for a single fitted MRV 


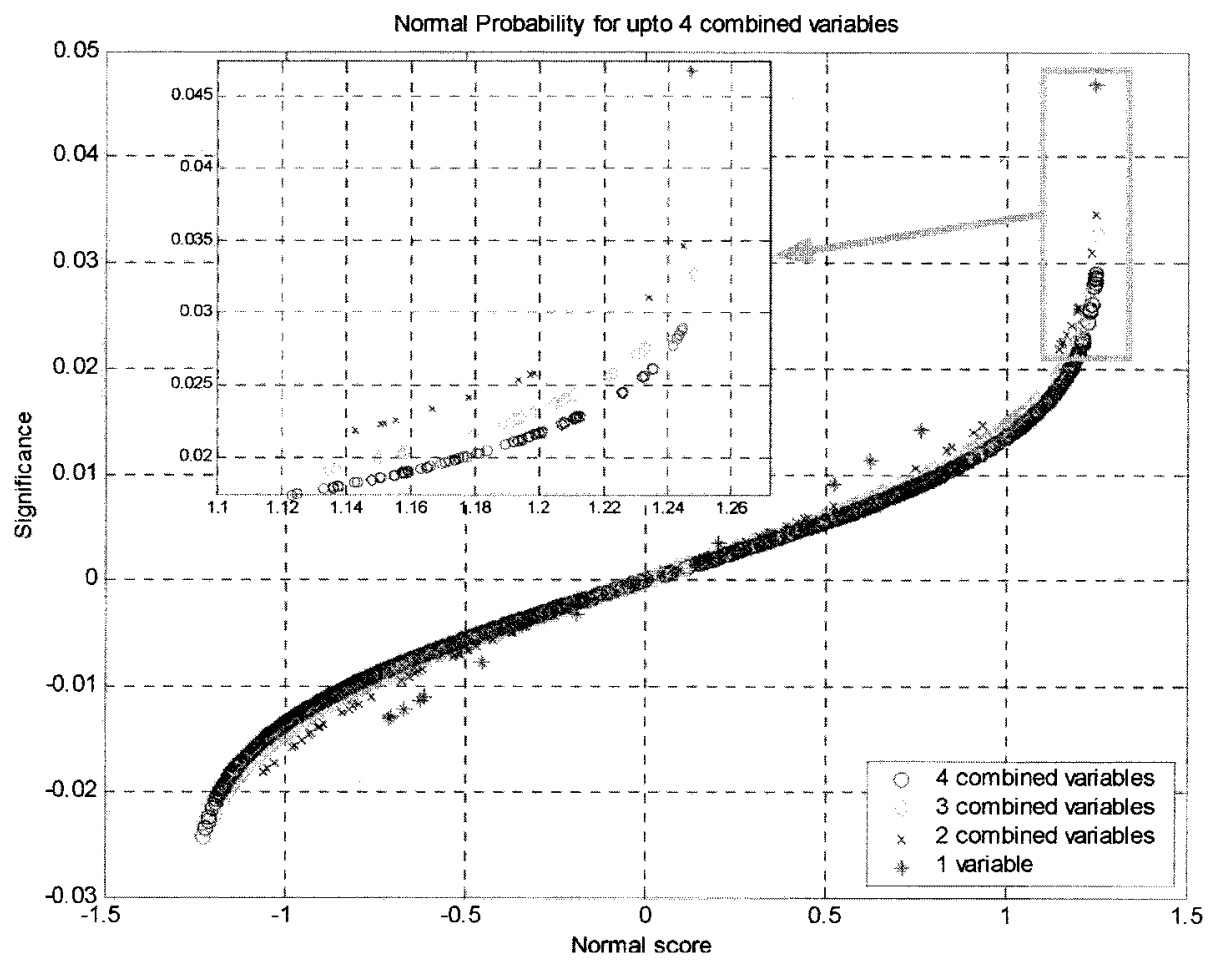

Fig. 5 Normal score plot for final factorial experiment

magnitude are considered further. Note that, in the figure, models with fewer MRVs appear more significant; these are not more accurate, however, as the plot omits the mean level of significance, which is lower for a lower number of MRVs. It should also be noted that the use of more than four MRVs in the CBM will result in a better fitting model, but that the overall statistical significance would be reduced. The 'perfect' model could be generated if enough parameters were used, but this would be a model that is a 'forced' fit of the particular test data seen here and not a general reflection of customer behaviour.

\subsection{Final customer behaviour model}

Results of the factorial experiment show that the CBM for pedal gain should take the form

$$
P=\alpha f_{1}+\beta f_{2}+\gamma f_{3}+\delta f_{4}+\varepsilon
$$

where the parameters $f_{1}, f_{2}, f_{3}$ and $f_{4}$ are defined by the factorial experiment and the coefficients $\alpha, \beta, \gamma, \delta$ and $\varepsilon$ are calculated using least-squares theory.

Figure 6a shows the final behaviour model regression, which exhibits a 95 per cent correlation between the 'ideal' progressions and the resultant model. Although none of the 'ideal' progressions is met exactly, 11 of the 12 candidates are within $P=0.2$ of their IIP. In general, candidates were found to be insensitive to a small change in progression; therefore, the model is considered acceptable. Figure $6 \mathrm{~b}$ shows the influence of each parameter on the final result; this shows the relatively even significance of each parameter in the model. The definition of a suitable CBM now allows the implementation of a prototype auto-adaptive electronic throttle control algorithm.

\section{APPLYING THE MODEL TO ON-LINE ADAPTIVE THROTTLE CONTROL}

Figure 6a highlights that, although the general fit of the model is good, allowing the model to run directly on the vehicle would lead to rapid local changes in pedal progression. The final output of the pedal gain is thus controlled by a first-order low-pass digital filter. This smooths out local variations and also allows the rate of adaptation to be controlled. Defining the general system as

$$
H(s)=\frac{a s+b}{c s+d}
$$

the coefficients are set at a suitable level such that rate control and noise filtering are optimal. To ensure that the system adapts to the correct level [i.e. steady state value $(\mathrm{SSV})=1]$, the final value theorem is applied. In this case $a=0, c=1$, and $b$ and $d$ are equal, defined to give a slow response rate.

To define the filter in a suitable format, such that $Y(k)$ can be obtained, the inverse Laplace transform of the ' $z$ transform' (applied using the 'Tustin' or trapezoidal 
(a) Final Customer Behaviour Model

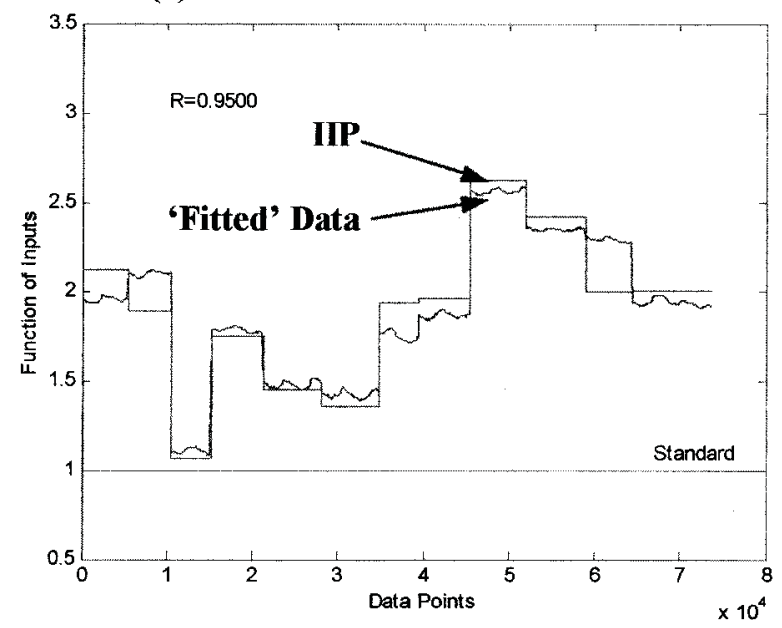

(b) Contribution of Individual Variables

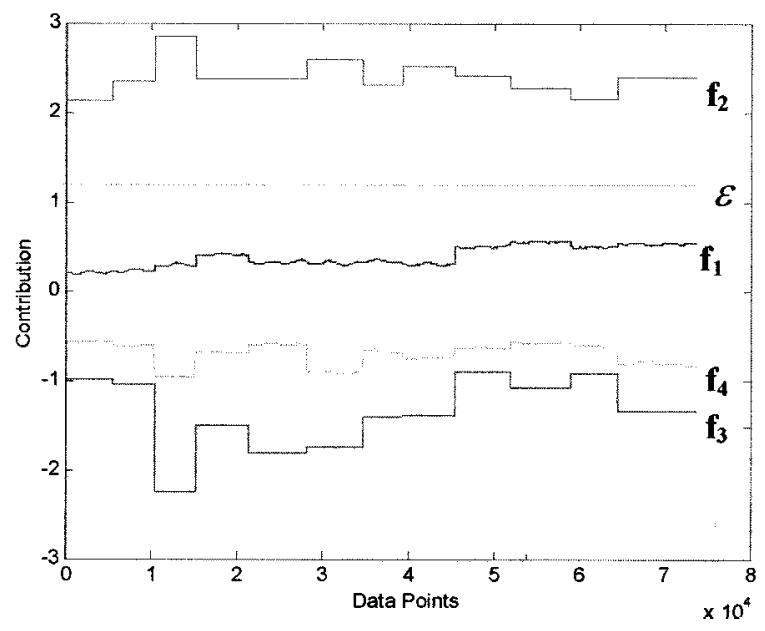

Fig. 6 Final CBM fit for all candidates, and significance of individual parameters

approach [4]) is taken; thus, the final filter has the form

$$
Y(k)=\frac{\hat{a} U(k)+\hat{b} U(k-1)-\hat{d} Y(k-1)}{\hat{c}}
$$

Only slow adaptation rates have been considered thus far, to allow sufficient analysis of the system to be carried out, and to respect safety issues before extensive analysis of the adaptation rate has been completed.

\subsection{Off-line results}

Figure 7 shows the result of a simulation of the adaptive system, using data recorded during the eye-test appraisal. A start point of $P=1.5$ has been used for the simulation. The final adapted value correlates well with IIP $=1.993$. The gain is not held constant for the full simulation of the drive, as the behaviour may change over time. The result signifies that the algorithm is suitable for field testing.

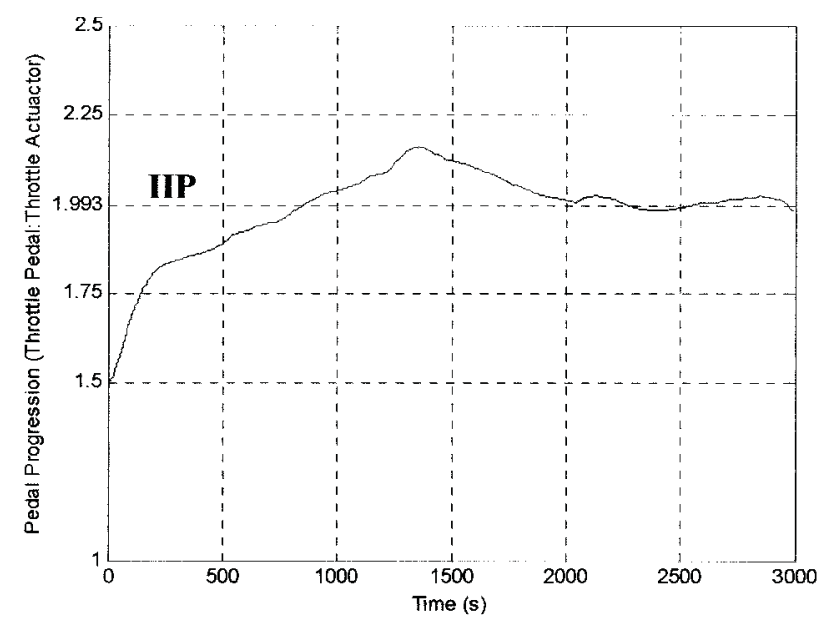

Fig. 7 Adaptation simulation of an eye test candidate

\subsection{On-line results}

Figure 8 shows that there is good correlation between the direction of adaptation and IIP, $P=1.352$ in an on-line implementation. The IIP found in the eye-test appraisal is not necessarily correct for that driver on the day of testing, it was only correct at the time of the eye test. IIP is dependent on influential factors on driving style, such as driving conditions, traffic, driver mood and time of day. Indication that the process was adapting to a suitable level was given by the fact that the driver was happier with the vehicle at the end of the driving period than at the beginning.

\section{CONCLUSIONS}

A novel on-line procedure has been developed to identify optimum or 'ideal' progression for any driver (the IIP). This procedure is a paired comparison technique labelled

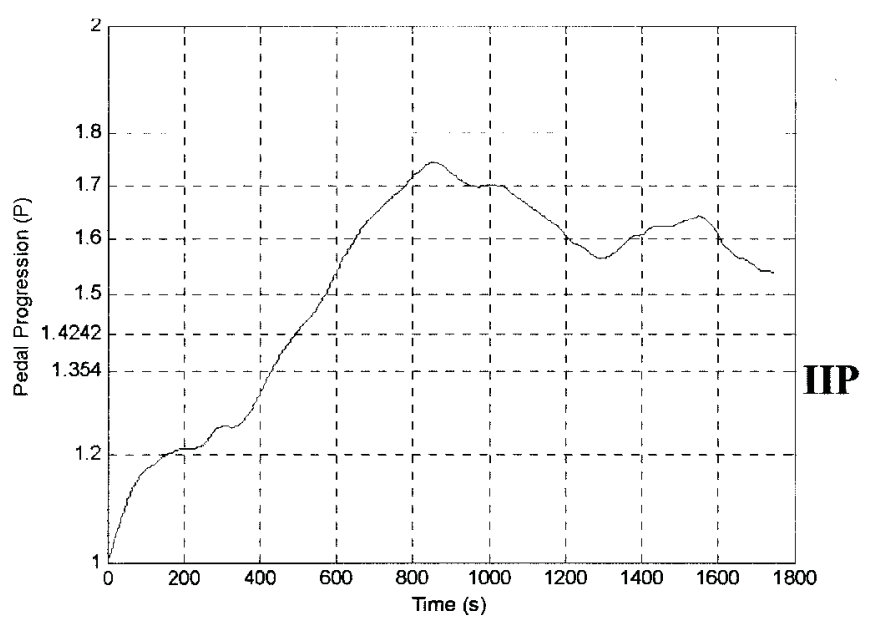

Fig. 8 Time history of pedal gain during a field trial 
the eye test. The procedure has developed a number of significant conclusions:

1. The novel methodology provides a reliable robust method for obtaining customer IIPs.

2. The eye-test method provides a useful tool through which a vehicle can be tailored to particular customer needs, by providing initial set-up values of pedal progression.

3. Candidates were found to be insensitive to small changes in progression.

4. Results from the eye-test appraisal indicate that drivers considered subjectively to be more aggressive in nature preferred a lower IIP. This indicates that they prefer and or require more control over the available power.

The formal statistical analysis procedure used to identify a CBM has produced the following conclusions:

1. The identified CBM gave a 95 per cent linear correlation between the 'ideal' progression and the resulting model.

2. Using many MRVs in the CBM will result in a better fitting model, but the overall statistical significance would be reduced.

3. Preliminary implementation of the model on a vehicle in real time demonstrates that the throttle progression adapts appropriately towards IIP, thereby matching the vehicle performance feel to driver expectations.

\section{ACKNOWLEDGEMENTS}

The authors would like to extend their gratitude to Ford Motor Company for giving permission to publish this paper and their support of the work throughout. Thanks are due to Adrain Broster for his work on the maintenance of the test vehicle and drive by wire system. Dr Mark Howell gave assistance during the development of the $\mathrm{C}$ code, for implementation on the Focus test vehicle, to whom sincere thanks are expressed.

\section{REFERENCES}

1 Passmore, M. A. A factorial study of performance feel: factors, effects and interactions. Report TT95R06, Department of Aeronautical and Automotive Engineering and Ford Motor Company, Loughborough University, 1995.

2 Passmore, M. A., Patel, A. and Lorentzen, R. The influence of engine demand map design on perceived performance. Int. J. Veh. Des., 2001, 26(5), 509-522.

3 Best, M. C. On the modelling requirements for the practical implementation of advanced vehicle suspension control. Doctoral thesis, Loughborough University, Loughborough, Leicestershire, 1995.

4 Franklin, G. F., Powell, J. D. and Workman, M. L. Digital Control of Dynamic Systems, 2nd edition, 1990 (AddisonWesley, Reading, Massachusetts).
5 Fleming, M. C. and Nellis, J. G. Principles of Applied Statistics, 1st edition, 1994 (Routledge, New York).

\section{APPENDIX}

\section{5th percentile $Q_{3}$}

Percentiles are a measure of the distribution of the data. They focus on the central proportion of the data excluding the influence of outliers. The position of the upper quartile $Q_{3}$ is expressed as

$$
Q_{3 \mathrm{POSN}}=\frac{3(n+1)}{4}
$$

where $n$ is the number of data points being sampled [5]. The $Q_{3}$ used is a rolling range parameter, being defined over a period of time. This lowers the variability and builds a true long-term model of the driver. The variability reductions compensate for ineffectual data such as that recorded while stationary at junctions, where both pedal position and vehicle speed are zero. The method used for calculating $Q_{3}$ is a 'box' technique. Calculation of an exact $Q_{3}$ would require the storage and sorting of an entire data set, which is inherently processor intensive and thus incapable of being executed in real time. The 'box' method is computationally much more efficient, allowing $Q_{3}$ to be found from a large data set which is continuously updated. Each 'box' is defined as a subdivision of the full range; thus

$$
\begin{aligned}
& b_{0}=X_{\mathrm{MIN}}: X_{\mathrm{MIN}}+(k+1) \\
& b_{1}=X_{\mathrm{MIN}}+(k+0.01): X_{\mathrm{MIN}}+(k+1) \\
& \vdots \\
& b_{k}=X_{\mathrm{MIN}}+(k+0.01): X_{\mathrm{MAX}}
\end{aligned}
$$

where

$$
\begin{aligned}
& X_{\mathrm{MIN}}=\text { lower limit of range } \\
& X_{\mathrm{MAX}}=\text { upper limit of range } \\
& k=\text { number of subdivisions } \\
& b=\text { subdivision of range }
\end{aligned}
$$

Each new data sample recorded is rounded up to its nearest integer and placed in its corresponding box. The 'first' data point from the stored range is then removed from the sample, allowing the range to move in time. The number of counts is recorded for each box, and the position of $Q_{3}$ is calculated thus:

$$
Q_{3 \mathrm{POSN}}=3\left(\sum_{i=k_{\mathrm{LO}}}^{i=k_{\mathrm{HI}}} x_{i}+1\right) / 4
$$

$Q_{3}$ is therefore the integer value of the box that contains $Q_{3 P O S N}$ :

$$
Q_{3}=b_{k}\left(x_{Q_{3 \mathrm{POSN}}}\right)
$$

The accuracy of the returned $Q_{3}$ is $-0.99+0.0$ units of 
the appropriate sampled variable. The use of these slowly varying parameters gives a baseline for the adaptation process to work from. Using a static $Q_{3}$ would cause a delay the same length of time to that over which the percentile is taken. This means that the adaptation process would always be adapting to how you have been driving and not how you are driving. This cannot be avoided, but its effects can be minimized. 